CUBO A Mathematical Journal Vol.16, $N^{0}$ 03, (21-35). October 2014

\title{
Higher Order Multivariate Fuzzy Approximation by basic Neural Network Operators
}

\author{
George A. Anastassiou \\ Department of Mathematical Sciences, \\ University of Memphis, Memphis, TN 38152, U.S.A. \\ ganastss@memphis.edu
}

\begin{abstract}
Here are studied in terms of multivariate fuzzy high approximation to the multivariate unit basic sequences of multivariate fuzzy neural network operators. These operators are multivariate fuzzy analogs of earlier studied multivariate real ones. The produced results generalize earlier real ones into the fuzzy setting. Here the high order multivariate fuzzy pointwise convergence with rates to the multivariate fuzzy unit operator is established through multivariate fuzzy inequalities involving the multivariate fuzzy moduli of continuity of the $\mathrm{Nth}$ order $(\mathrm{N} \geq 1) \mathrm{H}$-fuzzy partial derivatives, of the engaged multivariate fuzzy number valued function.
\end{abstract}

\section{RESUMEN}

Utilizando aproximaciones multivariadas difusas superiores, estudiamos la aplicación a secuencias básicas unitarias multivariadas de operadores de redes neuronales disfusas multivariadas. Estos operadores son análogos difusos multivariados de los reales multivariados estudiados anteriormente. Los resultados obtenidos generalizan los resultados reales anteriores en el marco difuso. La convergencia puntual difusa multivariada de orden superior con velocidades para los operadores unitarios difusos multivariados se establece a través de desigualdades difusas multivariadas que involucran los módulos de continuidad difusos multivariados de las derivadas parciales $\mathrm{H}$-difusas de $\mathrm{N}$-ésimo orden $(\mathrm{N} \geq 1)$ de las funciones con valores numéricos difusos multivariados.

Keywords and Phrases: multivariate fuzzy real analysis, multivariate fuzzy neural network operators, high order multivariate fuzzy approximation, multivariate fuzzy modulus of continuity and multivariate Jackson type inequalities.

2010 AMS Mathematics Subject Classification: 26A15, 26E50, 41A17, 41A25, 41A99, $47 \mathrm{~S} 40$. 


\section{Fuzzy real Analysis Background}

We need the following background

Definition 1. (see [14]) Let $\mu: \mathbb{R} \rightarrow[0,1]$ with the following properties

(i) is normal, i.e., $\exists x_{0} \in \mathbb{R} ; \mu\left(x_{0}\right)=1$.

(ii) $\mu(\lambda x+(1-\lambda) y) \geq \min \{\mu(x), \mu(y)\}, \forall x, y \in \mathbb{R}, \forall \lambda \in[0,1]$ ( $\mu$ is called a convex fuzzy subset).

(iii) $\mu$ is upper semicontinuous on $\mathbb{R}$, i.e. $\forall x_{0} \in \mathbb{R}$ and $\forall \varepsilon>0, \exists$ neighborhood $\mathrm{V}\left(\mathrm{x}_{0}\right)$ : $\mu(x) \leq \mu\left(x_{0}\right)+\varepsilon, \forall x \in V\left(x_{0}\right)$.

(iv) The set $\overline{\sup p(\mu)}$ is compact in $\mathbb{R}$, (where $\sup p(\mu):=\{x \in \mathbb{R}: \mu(x)>0\}$ ).

We call $\mu$ a fuzzy real number. Denote the set of all $\mu$ with $\mathbb{R}_{\mathcal{F}}$.

E.g. $\chi_{\left\{x_{0}\right\}} \in \mathbb{R}_{\mathcal{F}}$, for any $\chi_{0} \in \mathbb{R}$, where $\chi_{\left\{\chi_{0}\right\}}$ is the characteristic function at $\chi_{0}$.

For $0<r \leq 1$ and $\mu \in \mathbb{R}_{\mathcal{F}}$ define

$$
[\mu]^{r}:=\{x \in \mathbb{R}: \mu(x) \geq r\}
$$

and

$$
[\mu]^{0}:=\overline{\{x \in \mathbb{R}: \mu(x) \geq 0\}}
$$

Then it is well known that for each $r \in[0,1],[\mu]^{r}$ is a closed and bounded interval on $\mathbb{R}([11])$.

For $u, v \in \mathbb{R}_{\mathcal{F}}$ and $\lambda \in \mathbb{R}$, we define uniquely the sum $u \oplus v$ and the product $\lambda \odot u$ by

$$
[u \oplus v]^{r}=[u]^{r}+[v]^{r},[\lambda \odot u]^{r}=\lambda[u]^{r}, \forall r \in[0,1]
$$

where $[u]^{r}+[v]^{r}$ means the usual addition of two intervals (as subsets of $\mathbb{R}$ ) and $\lambda[u]^{r}$ means the usual product between a scalar and a subset of $\mathbb{R}$ (see, e.g. [14]).

Notice $1 \odot u=u$ and it holds

$$
\mathfrak{u} \oplus \boldsymbol{v}=\boldsymbol{v} \oplus \mathbf{u}, \lambda \odot \mathbf{u}=\mathfrak{u} \odot \lambda
$$

If $0 \leq r_{1} \leq r_{2} \leq 1$ then

$$
[u]^{r_{2}} \subseteq[u]^{r_{1}} .
$$

Actually $[u]^{r}=\left[u_{-}^{(r)}, u_{+}^{(r)}\right]$, where $u_{-}^{(r)} \leq u_{+}^{(r)}, u_{-}^{(r)}, u_{+}^{(r)} \in \mathbb{R}, \forall r \in[0,1]$.

For $\lambda>0$ one has $\lambda u_{ \pm}^{(r)}=(\lambda \odot u)_{ \pm}^{(r)}$, respectively.

Define D : $\mathbb{R}_{\mathcal{F}} \times \mathbb{R}_{\mathcal{F}} \rightarrow \mathbb{R}_{\mathcal{F}}$ by

$$
\mathrm{D}(\mathrm{u}, v):=\sup _{\mathrm{r} \in[0,1]} \max \left\{\left|\mathrm{u}_{-}^{(\mathrm{r})}-v_{-}^{(\mathrm{r})}\right|,\left|\mathrm{u}_{+}^{(\mathrm{r})}-v_{+}^{(\mathrm{r})}\right|\right\}
$$


where

$$
[v]^{\mathrm{r}}=\left[v_{-}^{(\mathrm{r})}, v_{+}^{(\mathrm{r})}\right] ; \mathrm{u}, v \in \mathbb{R}_{\mathcal{F}}
$$

We have that $\mathrm{D}$ is a metric on $\mathbb{R}_{\mathcal{F}}$.

Then $\left(\mathbb{R}_{\mathcal{F}}, \mathrm{D}\right)$ is a complete metric space, see [14, [15].

Let $f, g: \mathbb{R}^{m} \rightarrow \mathbb{R}_{\mathcal{F}}$. We define the distance

$$
D^{*}(f, g)=\sup _{x \in \mathbb{R}^{m}} D(f(x), g(x))
$$

Here $\Sigma^{*}$ stands for fuzzy summation and $\widetilde{0}:=\chi_{\{0\}} \in \mathbb{R}_{\mathcal{F}}$ is the neutral element with respect to $\oplus$, i.e.,

$$
u \oplus \widetilde{0}=\widetilde{0} \oplus u=u, \forall u \in \mathbb{R}_{\mathcal{F}} .
$$

We need

Remark 2. ([5]). Here $r \in[0,1], x_{i}^{(r)}, y_{i}^{(r)} \in \mathbb{R}, i=1, \ldots, m \in \mathbb{N}$. Suppose that

$$
\sup _{r \in[0,1]} \max \left(x_{i}^{(r)}, y_{i}^{(r)}\right) \in \mathbb{R}, \text { for } i=1, \ldots, m \text {. }
$$

Then one sees easily that

$$
\sup _{r \in[0,1]} \max \left(\sum_{i=1}^{m} x_{i}^{(r)}, \sum_{i=1}^{m} y_{i}^{(r)}\right) \leq \sum_{i=1}^{m} \sup _{r \in[0,1]} \max \left(x_{i}^{(r)}, y_{i}^{(r)}\right) .
$$

Definition 3. Let $\mathrm{f} \in \mathrm{C}\left(\mathbb{R}^{\mathrm{m}}\right), \mathrm{m} \in \mathbb{N}$, which is bounded or uniformly continuous, we define $(h>0)$

$$
\omega_{1}(f, h):=\sup _{\text {all } x_{i}, x_{i}^{\prime} \in \mathbb{R},\left|x_{i}-x_{i}^{\prime}\right| \leq h, \text { for } i=1, \ldots, m}\left|f\left(x_{1}, \ldots, x_{m}\right)-f\left(x_{1}^{\prime}, \ldots, x_{m}^{\prime}\right)\right| .
$$

Definition 4. Let $\mathrm{f}: \mathbb{R}^{\mathrm{m}} \rightarrow \mathbb{R}_{\mathcal{F}}$, we define the fuzzy modulus of continuity of $\mathrm{f}$ by

$$
\omega_{1}^{(\mathcal{F})}(f, \delta)=\sup _{x, y \in \mathbb{R},\left|x_{i}-y_{i}\right| \leq \delta, \text { for } i=1, \ldots, m} D(f(x), f(y)), \delta>0
$$

where $x=\left(x_{1}, \ldots, x_{m}\right), y=\left(y_{1}, \ldots, y_{m}\right)$.

For $\mathrm{f}: \mathbb{R}^{\mathrm{m}} \rightarrow \mathbb{R}_{\mathcal{F}}$, we use

$$
[\mathrm{f}]^{\mathrm{r}}=\left[\mathrm{f}_{-}^{(\mathrm{r})}, \mathrm{f}_{+}^{(\mathrm{r})}\right]
$$

where $\mathrm{f}_{ \pm}^{(\mathrm{r})}: \mathbb{R}^{\mathrm{m}} \rightarrow \mathbb{R}, \forall \mathrm{r} \in[0,1]$.

We need 
Proposition 5. Let $\mathrm{f}: \mathbb{R}^{\mathrm{m}} \rightarrow \mathbb{R}_{\mathcal{F}}$. Assume that $\omega_{1}^{\mathcal{F}}(\mathrm{f}, \delta), \omega_{1}\left(\mathrm{f}_{-}^{(\mathrm{r})}, \delta\right)$, $\omega_{1}\left(f_{+}^{(r)}, \delta\right)$ are finite for any $\delta>0, r \in[0,1]$.

Then

$$
\omega_{1}^{(\mathcal{F})}(f, \delta)=\sup _{r \in[0,1]} \max \left\{\omega_{1}\left(f_{-}^{(r)}, \delta\right), \omega_{1}\left(f_{+}^{(r)}, \delta\right)\right\}
$$

Proof. By Proposition 1 of $[8]$.

We define by $C_{\mathcal{F}}^{\mathrm{u}}\left(\mathbb{R}^{\mathrm{m}}\right)$ the space of fuzzy uniformly continuous functions from $\mathbb{R}^{\mathrm{m}} \rightarrow \mathbb{R}_{\mathcal{F}}$, also $C_{\mathcal{F}}\left(\mathbb{R}^{m}\right)$ is the space of fuzzy continuous functions on $\mathbb{R}^{m}$, and $C_{b}\left(\mathbb{R}^{m}, \mathbb{R}_{\mathcal{F}}\right)$ is the fuzzy continuous and bounded functions.

We mention

Proposition 6. ([7]) Let $\mathrm{f} \in \mathrm{C}_{\mathcal{F}}^{\mathrm{U}}\left(\mathbb{R}^{\mathrm{m}}\right)$. Then $\omega_{1}^{(\mathcal{F})}(\mathrm{f}, \delta)<\infty$, for any $\delta>0$.

Proposition 7. ([7]) It holds

$$
\lim _{\delta \rightarrow 0} \omega_{1}^{(\mathcal{F})}(f, \delta)=\omega_{1}^{(\mathcal{F})}(f, 0)=0
$$

iff $f \in \mathrm{C}_{\mathcal{F}}^{\mathrm{U}}\left(\mathbb{R}^{\mathrm{m}}\right)$.

Proposition 8. ([7]) Let $\mathrm{f} \in \mathrm{C}_{\mathcal{F}}\left(\mathbb{R}^{\mathrm{m}}\right)$. Then $\mathrm{f}_{ \pm}^{(\mathrm{r})}$ are equicontinuous with respect to $\mathrm{r} \in[0,1]$ over $\mathbb{R}^{m}$, respectively in \pm .

Note: It is clear by Propositions 囵, 雨, that if $\mathrm{f} \in \mathrm{C}_{\mathcal{F}}^{\mathrm{U}}\left(\mathbb{R}^{\mathrm{m}}\right)$, then $\mathrm{f}_{ \pm}^{(\mathrm{r})} \in \mathrm{C}_{\mathrm{u}}\left(\mathbb{R}^{\mathrm{m}}\right)$ (uniformly continuous on $\mathbb{R}^{\mathrm{m}}$ ).

We need

Definition 9. Let $x, y \in \mathbb{R}_{\mathcal{F}}$. If there exists $z \in \mathbb{R}_{\mathcal{F}}: x=y \oplus z$, then we call $z$ the $\mathrm{H}$-difference on $\mathrm{x}$ and $\mathrm{y}$, denoted $\mathrm{x}-\mathrm{y}$.

Definition 10. ([14]) Let $\mathrm{T}:=\left[\mathrm{x}_{0}, \mathrm{x}_{0}+\beta\right] \subset \mathbb{R}$, with $\beta>0$. A function $\mathrm{f}: \mathrm{T} \rightarrow \mathbb{R}_{\mathcal{F}}$ is $\mathrm{H}$-difference at $\mathrm{x} \in \mathrm{T}$ if there exists an $\mathrm{f}^{\prime}(\mathrm{x}) \in \mathbb{R}_{\mathcal{F}}$ such that the limits (with respect to $\mathrm{D}$ )

$$
\lim _{h \rightarrow 0+} \frac{f(x+h)-f(x)}{h}, \lim _{h \rightarrow 0+} \frac{f(x)-f(x-h)}{h}
$$

exist and are equal to $\mathrm{f}^{\prime}(\mathrm{x})$.

We call $\mathrm{f}^{\prime}$ the $\mathrm{H}$-derivative or fuzzy derivative of $\mathrm{f}$ at $\mathrm{x}$.

Above is assumed that the H-differences $f(x+h)-f(x), f(x)-f(x-h)$ exists in $\mathbb{R}_{\mathcal{F}}$ in a neighborhood of $x$.

Definition 11. We denote by $\mathrm{C}_{\mathcal{F}}^{N}\left(\mathbb{R}^{\mathrm{m}}\right), \mathrm{N} \in \mathbb{N}$, the space of all $\mathrm{N}$-times fuzzy continuously differentiable functions from $\mathbb{R}^{\mathrm{m}}$ into $\mathbb{R}_{\mathcal{F}}$. 
Here fuzzy partial derivatives are defined via Definition[10 in the obvious way as in the ordinary real case.

We mention

Theorem 12. ([12]) Let $\mathrm{f}:[\mathrm{a}, \mathrm{b}] \subseteq \mathbb{R} \rightarrow \mathbb{R}_{\mathcal{F}}$ be $\mathrm{H}$-fuzzy differentiable. Let $\mathrm{t} \in[\mathrm{a}, \mathrm{b}], 0 \leq \mathrm{r} \leq 1$. Clearly

$$
[f(t)]^{r}=\left[f(t)_{-}^{(r)}, f(t)_{+}^{(r)}\right] \subseteq \mathbb{R} .
$$

Then $(\mathrm{f}(\mathrm{t}))_{ \pm}^{(\mathrm{r})}$ are differentiable and

$$
\left[f^{\prime}(t)\right]^{r}=\left[\left(f(t)_{-}^{(r)}\right)^{\prime},\left(f(t)_{+}^{(r)}\right)^{\prime}\right]
$$

I.e.

$$
\left(\mathrm{f}^{\prime}\right)_{ \pm}^{(\mathrm{r})}=\left(\mathrm{f}_{ \pm}^{(\mathrm{r})}\right)^{\prime}, \quad \forall \mathrm{r} \in[0,1] .
$$

Remark 13. (se also [6]) Let $\mathrm{f} \in \mathrm{C}^{\mathrm{N}}\left(\mathbb{R}, \mathbb{R}_{\mathcal{F}}\right), \mathrm{N} \geq 1$. Then by Theorem 10 we obtain $\mathrm{f}_{ \pm}^{(\mathrm{r})} \in$ $\mathrm{C}^{\mathrm{N}}(\mathbb{R})$ and

$$
\left[f^{(i)}(t)\right]^{r}=\left[\left(f(t)_{-}^{(r)}\right)^{(i)},\left(f(t)_{+}^{(r)}\right)^{(i)}\right]
$$

for $\mathrm{i}=0,1,2, \ldots, \mathrm{N}$, and in particular we have

$$
\left(f^{(i)}\right)_{ \pm}^{(r)}=\left(f_{ \pm}^{(r)}\right)^{(i)}
$$

for any $r \in[0,1]$.

Let $f \in C_{\mathcal{F}}^{N}\left(\mathbb{R}^{m}\right)$, denote $f_{\widetilde{\alpha}}:=\frac{\partial^{\tilde{\alpha} f}}{\partial x^{\alpha}}$, where $\widetilde{\alpha}:=\left(\widetilde{\alpha_{1}}, \ldots, \widetilde{\alpha_{m}}\right), \widetilde{\alpha_{i}} \in \mathbb{Z}^{+}, i=1, \ldots, m$ and

$$
0<|\widetilde{\alpha}|:=\sum_{i=1}^{m} \widetilde{\alpha_{i}} \leq N, \quad N>1
$$

Then by Theorem 12 we get that

$$
\left(f_{ \pm}^{(r)}\right)_{\widetilde{\alpha}}=\left(f_{\widetilde{\alpha}}\right)_{ \pm}^{(r)}, \forall r \in[0,1]
$$

and any $\widetilde{\alpha}:|\widetilde{\alpha}| \leq N$. Here $f_{ \pm}^{(r)} \in C^{N}\left(\mathbb{R}^{m}\right)$.

For the definition of general fuzzy integral we follow [13] next.

Definition 14. Let $(\Omega, \Sigma, \mu)$ be a complete $\sigma$-finite measure space. We call $\mathrm{F}: \Omega \rightarrow \mathbb{R}_{\mathcal{F}}$ measurable iff $\forall$ closed $\mathrm{B} \subseteq \mathbb{R}$ the function $\mathrm{F}^{-1}(\mathrm{~B}): \Omega \rightarrow[0,1]$ defined by

$$
\mathrm{F}^{-1}(\mathrm{~B})(w):=\sup _{\mathrm{x} \in \mathrm{B}} \mathrm{F}(w)(\mathrm{x}), \text { all } w \in \Omega
$$

is measurable, see [13]. 
Theorem 15. ([13]) For $\mathrm{F}: \Omega \rightarrow \mathbb{R}_{\mathcal{F}}$,

$$
F(w)=\left\{\left(F_{-}^{(r)}(w), F_{+}^{(r)}(w)\right) \mid 0 \leq r \leq 1\right\},
$$

the following are equivalent

(1) $\mathrm{F}$ is measurable,

(2) $\forall \mathrm{r} \in[0,1], \mathrm{F}_{-}^{(\mathrm{r})}, \mathrm{F}_{+}^{(\mathrm{r})}$ are measurable.

Following [13, given that for each $r \in[0,1], F_{-}^{(r)}, F_{+}^{(r)}$ are integrable we have that the parametrized representation

$$
\left\{\left(\int_{A} F_{-}^{(r)} \mathrm{d} \mu, \int_{A} F_{+}^{(r)} \mathrm{d} \mu\right) \mid 0 \leq r \leq 1\right\}
$$

is a fuzzy real number for each $A \in \Sigma$.

The last fact leads to

Definition 16. ([13]) A measurable function $\mathrm{F}: \Omega \rightarrow \mathbb{R}_{\mathcal{F}}$,

$$
\mathrm{F}(w)=\left\{\left(\mathrm{F}_{-}^{(\mathrm{r})}(w), \mathrm{F}_{+}^{(\mathrm{r})}(w)\right) \mid 0 \leq r \leq 1\right\}
$$

is integrable if for each $\mathrm{r} \in[0,1], \mathrm{F}_{ \pm}^{(\mathrm{r})}$ are integrable, or equivalently, if $\mathrm{F}_{ \pm}^{(0)}$ are integrable.

In this case, the fuzzy integral of $\mathrm{F}$ over $\mathrm{A} \in \Sigma$ is defined by

$$
\int_{A} F d \mu:=\left\{\left(\int_{A} F_{-}^{(r)} d \mu, \int_{A} F_{+}^{(r)} d \mu\right) \mid 0 \leq r \leq 1\right\} .
$$

By [13] $\mathrm{F}$ is integrable iff $w \rightarrow\|\mathrm{F}(w)\|_{\mathcal{F}}$ is real-valeud integrable. Here

$$
\|\mathrm{u}\|_{\mathcal{F}}:=\mathrm{D}(\mathrm{u}, \tilde{0}), \forall \mathrm{u} \in \mathbb{R}_{\mathcal{F}}
$$

We need also

Theorem 17. ([13]) Let $\mathrm{F}, \mathrm{G}: \Omega \rightarrow \mathbb{R}_{\mathcal{F}}$ be integrable. Then

(1) Let $\mathrm{a}, \mathrm{b} \in \mathbb{R}$, then $\mathrm{aF}+\mathrm{bG}$ is integrable and for each $A \in \Sigma$,

$$
\int_{A}(a F+b G) d \mu=a \int_{A} F d \mu+b \int_{A} G d \mu
$$

(2) $\mathrm{D}(\mathrm{F}, \mathrm{G})$ is a real-valued integrable function and for each $\mathrm{A} \in \Sigma$,

$$
D\left(\int_{A} F d \mu, \int_{A} G d \mu\right) \leq \int_{A} D(F, G) d \mu .
$$

In particular,

$$
\left\|\int_{A} F d \mu\right\|_{\mathcal{F}} \leq \int_{A}\|F\|_{\mathcal{F}} d \mu
$$


Above $\mu$ could be the Lebesgue measure, with all the basic properties valid here too.

Basically here we have

$$
\left[\int_{A} F d \mu\right]^{r}:=\left[\int_{A} F_{-}^{(r)} d \mu, \int_{A} F_{+}^{(r)} d \mu\right]
$$

i.e.

$$
\left(\int_{A} F d \mu\right)_{ \pm}^{(r)}=\int_{A} F_{ \pm}^{(r)} \mathrm{d} \mu
$$

$\forall \mathrm{r} \in[0,1]$, respectively.

We use

Notation 18. We denote

$$
\begin{gathered}
\left(\sum_{i=1}^{2} \mathrm{D}\left(\frac{\partial}{\partial x_{i}}, \tilde{0}\right)\right)^{2} \mathrm{f}(\vec{x}):= \\
\mathrm{D}\left(\frac{\partial^{2} \mathrm{f}\left(\mathrm{x}_{1}, x_{2}\right)}{\partial x_{1}^{2}}, \tilde{0}\right)+\mathrm{D}\left(\frac{\partial^{2} \mathrm{f}\left(\mathrm{x}_{1}, x_{2}\right)}{\partial x_{2}^{2}}, \tilde{0}\right)+2 \mathrm{D}\left(\frac{\partial^{2} \mathrm{f}\left(\mathrm{x}_{1}, x_{2}\right)}{\partial x_{1} \partial x_{2}}, \tilde{0}\right) .
\end{gathered}
$$

In general we denote $(\mathrm{j}=1, \ldots, \mathrm{N})$

$$
\begin{gathered}
\left(\sum_{i=1}^{m} D\left(\frac{\partial}{\partial x_{i}}, \tilde{0}\right)\right)^{j} f(\vec{x}):= \\
\sum_{\left(j_{1}, \ldots, j_{m}\right) \in \mathbb{Z}_{+}^{m}: \sum_{i=1}^{m} j_{i}=j} \frac{j !}{j_{1} ! j_{2} ! \ldots j_{m} !} D\left(\frac{\partial^{j} f\left(x_{1}, \ldots, x_{m}\right)}{\partial x_{1}^{j_{1}} \partial x_{2}^{j_{2}} \ldots \partial x_{m}^{j_{m}}}, \tilde{0}\right) .
\end{gathered}
$$

\section{Convergence with rates of real multivariate neural net- work operators}

Here we follow 9 .

We need the following (see [10]) definitions.

Definition 19. A function $\mathrm{b}: \mathbb{R} \rightarrow \mathbb{R}$ is said to be bell-shaped if $\mathrm{b}$ belongs to $\mathrm{L}^{1}$ and its integral is nonzero, if it is nondecreasing on $(-\infty, \mathrm{a})$ and nonincreasing on $[\mathrm{a},+\infty)$, where a belongs to $\mathbb{R}$. In particular $\mathrm{b}(\mathrm{x})$ is a nonnegative number and at $\mathrm{a}, \mathrm{b}$ takes a global maximum; it is the center of the bell-shaped function. A bell-shaped function is said to be centered if its center is zero.

Definition 20. (see [10]) A function $\mathrm{b}: \mathbb{R}^{\mathrm{d}} \rightarrow \mathbb{R}(\mathrm{d} \geq 1)$ is said to be a $\mathrm{d}$-dimensional bell-shaped function if it is integrable and its integral is not zero, and for all $\mathrm{i}=1, \ldots, \mathrm{d}$,

$$
t \rightarrow b\left(x_{1}, \ldots, t, \ldots, x_{d}\right)
$$

is a centered bell-shaped function, where $\vec{x}:=\left(x_{1}, \ldots, x_{\mathrm{d}}\right) \in \mathbb{R}^{\mathrm{d}}$ arbitrary. 
Example 21. (from [10]) Let $\mathrm{b}$ be a centered bell-shaped function over $\mathbb{R}$, then $\left(\mathrm{x}_{1}, \ldots, \mathrm{x}_{\mathrm{d}}\right) \rightarrow$ $\mathrm{b}\left(\mathrm{x}_{1}\right) \ldots \mathrm{b}\left(\mathrm{x}_{\mathrm{d}}\right)$ is a $\mathrm{d}$-dimensional bell-shaped function.

Assumption 22. Here $\mathrm{b}(\vec{x})$ is of compact support $\mathcal{B}:=\prod_{i=1}^{\mathrm{d}}\left[-\mathrm{T}_{i}, \mathrm{~T}_{i}\right], \mathrm{T}_{i}>0$ and it may have jump discontinuities there. Let $\mathrm{f}: \mathbb{R}^{\mathrm{d}} \rightarrow \mathbb{R}$ be a continuous and bounded function or a uniformly continuous function.

Here we mention the study ([9]) of poitwise convergence with rates over $\mathbb{R}^{\mathrm{d}}$, to the unit operator I, of the "normalized bell" real multivariate neural network operators

$$
\begin{gathered}
M_{n}(f)(\vec{x}):= \\
\frac{\sum_{k_{1}=-n^{2}}^{n^{2}} \ldots \sum_{k_{d}=-n^{2}}^{n^{2}} f\left(\frac{k_{1}}{n}, \ldots \frac{k_{d}}{n}\right) b\left(n^{1-\alpha}\left(x_{1}-\frac{k_{1}}{n}\right), \ldots, n^{1-\alpha}\left(x_{d}-\frac{k_{d}}{n}\right)\right)}{\sum_{k_{1}=-n^{2}}^{n^{2}} \cdots \sum_{k_{d}=-n^{2}}^{n^{2}} b\left(n^{1-\alpha}\left(x_{1}-\frac{k_{1}}{n}\right), \ldots, n^{1-\alpha}\left(x_{d}-\frac{k_{d}}{n}\right)\right)},
\end{gathered}
$$

where $0<\alpha<1$ and $\vec{x}:=\left(x_{1}, \ldots, x_{d}\right) \in \mathbb{R}^{\mathrm{d}}, \mathrm{n} \in \mathbb{N}$. Clearly, $\mathrm{M}_{\mathrm{n}}$ is a positive linear operator.

The terms in the ratio of multiple sums (19) can be nonzero iff simultaneously

$$
\left|n^{1-\alpha}\left(x_{i}-\frac{k_{i}}{n}\right)\right| \leq T_{i}, \text { all } i=1, \ldots, d,
$$

i.e., $\left|x_{i}-\frac{k_{i}}{n}\right| \leq \frac{T_{i}}{n^{1-\alpha}}$, all $i=1, \ldots, d$, iff

$$
n x_{i}-T_{i} n^{\alpha} \leq k_{i} \leq n x_{i}+T_{i} n^{\alpha}, \text { all } i=1, . ., d .
$$

To have the order

$$
-n^{2} \leq n x_{i}-T_{i} n^{\alpha} \leq k_{i} \leq n x_{i}+T_{i} n^{\alpha} \leq n^{2}
$$

we need $n \geq T_{i}+\left|x_{i}\right|$, all $i=1, . ., d$. So (21) is true when we take

$$
n \geq \max _{i \in\{1, \ldots, d\}}\left(T_{i}+\left|x_{i}\right|\right) .
$$

When $\vec{x} \in \mathcal{B}$ in order to have (21) it is enough to assume that $n \geq 2 T^{*}$, where $T^{*}:=\max \left\{T_{1}, \ldots, T_{d}\right\}>$ 0. Consider

$$
\widetilde{\mathrm{I}_{i}}:=\left[n x_{i}-\mathrm{T}_{i} n^{\alpha}, n x_{i}+\mathrm{T}_{i} n^{\alpha}\right], i=1, \ldots, d, n \in \mathbb{N} .
$$

The length of $\widetilde{I_{i}}$ is $2 T_{i} n^{\alpha}$. By Proposition 1 of [1, we get that the cardinality of $k_{i} \in \mathbb{Z}$ that belong to $\widetilde{I_{i}}:=\operatorname{card}\left(k_{i}\right) \geq \max \left(2 T_{i} n^{\alpha}-1,0\right)$, any $i \in\{1, \ldots, d\}$. In order to have card $\left(k_{i}\right) \geq 1$, we need $2 \mathrm{~T}_{i} \mathrm{n}^{\alpha}-1 \geq 1$ iff $\mathrm{n} \geq \mathrm{T}_{i}^{-\frac{1}{\alpha}}$, any $i \in\{1, \ldots, \mathrm{d}\}$.

Therefore, a sufficient condition in order to obtain the order (21) along with the interval $\widetilde{I}_{i}$ to contain at least one integer for all $i=1, \ldots, d$ is that

$$
n \geq \max _{i \in\{1, \ldots, d\}}\left\{T_{i}+\left|x_{i}\right|, T_{i}^{-\frac{1}{\alpha}}\right\} .
$$


Clearly as $n \rightarrow+\infty$ we get that $\operatorname{card}\left(k_{i}\right) \rightarrow+\infty$, all $i=1, \ldots, d$. Also notice that card $\left(k_{i}\right)$ equals to the cardinality of integers in $\left[\left[n x_{i}-T_{i} n^{\alpha}\right\rceil,\left[n x_{i}+T_{i} n^{\alpha}\right]\right]$ for all $i=1, \ldots, d$. Here, [.] denotes the integral part of the number, while $\lceil\cdot\rceil$ denotes its ceiling.

From now on, in this article we will assume (23). Furthermore it holds

$$
\begin{gathered}
\left(M_{n}(f)\right)(\vec{x}):=\frac{\sum_{k_{1}=\left\lceil n x_{1}-T_{1} n^{\alpha}\right\rceil \cdots \sum_{k_{d}=\left\lceil n x_{d}-T_{d} n^{\alpha}\right\rceil}^{\left[n x_{1}+T_{1} n^{\alpha}\right]} f\left(\frac{k_{1}}{n}, \ldots \frac{k_{d}}{n}\right)}^{[\vec{x})}}{\cdot b\left(n^{1-\alpha}\left(x_{1}-\frac{k_{1}}{n}\right), \ldots, n^{1-\alpha}\left(x_{d}-\frac{k_{d}}{n}\right)\right)}
\end{gathered}
$$

all $\vec{x}:=\left(x_{1}, \ldots, x_{d}\right) \in \mathbb{R}^{d}$, where

$$
\begin{gathered}
V(\vec{x}):= \\
\sum_{k_{1}=\left\lceil n x_{1}-T_{1} n^{\alpha}\right\rceil}^{\left[n x_{1}+T_{1} n^{\alpha}\right]} \ldots \sum_{k_{d}=\left\lceil n x_{d}-T_{d} n^{\alpha}\right\rceil}^{\left[n x_{d}+T_{d} n^{\alpha}\right]} b\left(n^{1-\alpha}\left(x_{1}-\frac{k_{1}}{n}\right), \ldots, n^{1-\alpha}\left(x_{d}-\frac{k_{d}}{n}\right)\right) .
\end{gathered}
$$

From [9], we need and mention

Theorem 23. Let $\vec{x} \in \mathbb{R}^{\mathrm{d}}$; then

$$
\left|\left(M_{n}(f)\right)(\vec{x})-f(\vec{x})\right| \leq w_{1}\left(f, \frac{T^{*}}{n^{1-\alpha}}\right) .
$$

Inequality (26) is attained by constant functions.

Inequalities (26) gives $M_{n}(f)(\vec{x}) \rightarrow f(\vec{x})$, pointwise with rates, as $\mathrm{n} \rightarrow+\infty$, where $\vec{\chi} \in \mathbb{R}^{\mathrm{d}}, \mathrm{d} \geq 1$, provided that $\mathrm{f}$ is uniformly continuous on $\mathbb{R}^{\mathrm{d}}$. In the last case it is clear that $\mathrm{M}_{\mathrm{n}} \rightarrow \mathrm{I}$, uniformly.

From [9], we also need and mention

Theorem 24. Let $\vec{x} \in \mathbb{R}^{\mathrm{d}}, \mathrm{f} \in \mathrm{C}^{\mathrm{N}}\left(\mathbb{R}^{\mathrm{d}}\right), \mathrm{N} \in \mathbb{N}$, such that all of its partial derivatives $\mathrm{f}_{\widetilde{\alpha}}$ of order $\mathrm{N}, \widetilde{\alpha}:|\widetilde{\alpha}|=\mathrm{N}$, are uniformly continuous or continuous are bounded. Then

$$
\begin{gathered}
\left|\left(M_{n}(f)\right)(\vec{x})-f(\vec{x})\right| \leq \\
\left\{\sum_{j=1}^{N} \frac{\left(T^{*}\right)^{j}}{j ! n^{j(1-\alpha)}}\left(\left(\sum_{i=1}^{d}\left|\frac{\partial}{\partial x_{i}}\right|\right)^{j} f(\vec{x})\right)\right\}+\frac{\left(T^{*}\right)^{N} d^{N}}{N ! n^{N(1-\alpha)}} \cdot \max _{\widetilde{\alpha}:|\widetilde{\alpha}|=N} \omega_{1}\left(f \widetilde{\alpha}, \frac{T^{*}}{n^{1-\alpha}}\right) .
\end{gathered}
$$

Inequality (27) is attained by constant functions. Also, (27) gives us with rates the pointwise convergences of $\mathrm{M}_{\mathrm{n}}(\mathrm{f}) \rightarrow \mathrm{f}$ over $\mathbb{R}^{\mathrm{d}}$, as $\mathrm{n} \rightarrow+\infty$. 


\section{Main Results - Convergence with rates of fuzzy multivari- ate neural networks}

Here $b$ is as in Definition 20 .

Assumption 25. We suppose that $\mathrm{b}(\overrightarrow{\mathrm{x}})$ is of compact support $\mathrm{B}:=\prod_{i=1}^{\mathrm{d}}\left[-\mathrm{T}_{i}, \mathrm{~T}_{i}\right], \mathrm{T}_{\mathrm{i}}>0$, and it may have jump discontinuities there. We consider $\mathrm{f}: \mathbb{R}^{\mathrm{d}} \rightarrow \mathbb{R}_{\mathcal{F}}$ to be fuzzy continuous and fuzzy bounded function or fuzzy uniformly continuous function.

In this section we study the D-metric pointwise convergence with rates over $\mathbb{R}^{\mathrm{d}}$, to the fuzzy unit operator $\mathrm{I}_{\mathcal{F}}$, of the fuzzy multivariate neural network operators $\left(0<\alpha<1, \vec{x}:=\left(x_{1}, \ldots, x_{d}\right) \in\right.$ $\left.\mathbb{R}^{\mathrm{d}}, n \in \mathbb{N}\right)$

$$
\begin{aligned}
& M_{n}^{\mathcal{F}}(f)(\vec{x}):= \\
& \frac{\sum_{k_{1}=-n^{2}}^{n^{2} *} \sum_{k_{d}=-n^{2}}^{n^{2} *} f\left(\frac{k_{1}}{n}, \ldots \frac{k_{d}}{n}\right) \odot b\left(n^{1-\alpha}\left(x_{1}-\frac{k_{1}}{n}\right), \ldots, n^{1-\alpha}\left(x_{d}-\frac{k_{d}}{n}\right)\right)}{\sum_{k_{1}=-n^{2}}^{n^{2}} \cdots \sum_{k_{d}=-n^{2}}^{n^{2}} b\left(n^{1-\alpha}\left(x_{1}-\frac{k_{1}}{n}\right), \ldots, n^{1-\alpha}\left(x_{d}-\frac{k_{d}}{n}\right)\right)} \\
& =\sum_{k_{1}=\left\lceil n x_{1}-T_{1} n^{\alpha}\right\rceil}^{\left[n x_{1}+T_{1} n^{\alpha}\right] *} \sum_{k_{d}=\left\lceil n x_{d}-T_{d} n^{\alpha}\right\rceil}^{\left[n x_{d}+T_{d} n^{\alpha}\right] *} f\left(\frac{k_{1}}{n}, \ldots \frac{k_{d}}{n}\right) \\
& \odot \frac{b\left(n^{1-\alpha}\left(x_{1}-\frac{k_{1}}{n}\right), \ldots, n^{1-\alpha}\left(x_{d}-\frac{k_{d}}{n}\right)\right)}{V(\vec{x})},
\end{aligned}
$$

where $V(\vec{x})$ as in (25) and under the assumption (23).

We notice for $r \in[0,1]$ that

$$
\begin{aligned}
& {\left[M_{n}^{\mathcal{F}}(f)(\vec{x})\right]^{r}=\sum_{k_{1}=\left\lceil n x_{1}-T_{1} n^{\alpha}\right\rceil}^{\left[n x_{1}+T_{1} n^{\alpha}\right]} \ldots \sum_{k_{d}=\left\lceil n x_{d}-T_{d} n^{\alpha}\right\rceil}^{\left[n x_{d}+T_{d} n^{\alpha}\right]}\left[f\left(\frac{k_{1}}{n}, \ldots \frac{k_{d}}{n}\right)\right]^{r}} \\
& \frac{b\left(n^{1-\alpha}\left(x_{1}-\frac{k_{1}}{n}\right), \ldots, n^{1-\alpha}\left(x_{d}-\frac{k_{d}}{n}\right)\right)}{V(\vec{x})} \\
& =\sum_{k_{1}=\left\lceil n x_{1}-T_{1} n^{\alpha}\right\rceil}^{\left[n x_{1}+T_{1} n^{\alpha}\right]} \ldots \sum_{k_{d}=\left\lceil n x_{d}-T_{d} n^{\alpha}\right\rceil}^{\left[n x_{d}+T_{d} n^{\alpha}\right]}\left[f_{-}^{(r)}\left(\frac{k_{1}}{n}, \ldots \frac{k_{d}}{n}\right), f_{+}^{(r)}\left(\frac{k_{1}}{n}, \ldots \frac{k_{d}}{n}\right)\right] \\
& \frac{b\left(n^{1-\alpha}\left(x_{1}-\frac{k_{1}}{n}\right), \ldots, n^{1-\alpha}\left(x_{d}-\frac{k_{d}}{n}\right)\right)}{V(\vec{x})} \\
& =\left[\sum_{k_{1}=\left\lceil n x_{1}-T_{1} n^{\alpha}\right\rceil}^{\left[n x_{1}+T_{1} n^{\alpha}\right]} \ldots \sum_{k_{d}=\left\lceil n x_{d}-T_{d} n^{\alpha}\right\rceil}^{\left[n x_{d}+T_{d} n^{\alpha}\right]} f_{-}^{(r)}\left(\frac{k_{1}}{n}, \ldots \frac{k_{d}}{n}\right)\right.
\end{aligned}
$$




$$
\begin{gathered}
\cdot \frac{b\left(n^{1-\alpha}\left(x_{1}-\frac{k_{1}}{n}\right), \ldots, n^{1-\alpha}\left(x_{d}-\frac{k_{d}}{n}\right)\right)}{v(\vec{x})}, \\
\sum_{k_{1}=\left\lceil n x_{1}-T_{1} n^{\alpha}\right\rceil}^{\left[n x_{1}+T_{1} n^{\alpha}\right]} \sum_{k_{d}=\left\lceil n x_{d}-T_{d} n^{\alpha}\right\rceil}^{\left[n x_{d}+T_{d} n^{\alpha}\right]} f_{+}^{(r)}\left(\frac{k_{1}}{n}, \ldots \frac{k_{d}}{n}\right) \\
\left.=\frac{b\left(n^{1-\alpha}\left(x_{1}-\frac{k_{1}}{n}\right), \ldots, n^{1-\alpha}\left(x_{d}-\frac{k_{d}}{n}\right)\right)}{v(\vec{x})}\right] \\
=\left[\left(M_{n}\left(f_{-}^{(r)}\right)\right)(\vec{x}),\left(M_{n}\left(f_{+}^{(r)}\right)\right)(\vec{x})\right] .
\end{gathered}
$$

We have proved that

$$
\left(M_{\mathfrak{n}}^{\mathcal{F}}(f)\right)_{ \pm}^{(r)}=M_{n}\left(f_{ \pm}^{(r)}\right), \quad \forall r \in[0,1]
$$

respectively.

We present

Theorem 26. Let $\vec{x} \in \mathbb{R}^{\mathrm{d}}$; then

$$
\mathrm{D}\left(\left(M_{\mathfrak{n}}^{\mathcal{F}}(f)\right)(\vec{x}), f(\vec{x})\right) \leq \omega_{1}^{(\mathcal{F})}\left(f, \frac{T^{*}}{n^{1-\alpha}}\right) .
$$

Notice that (33) gives $\mathrm{M}_{\mathrm{n}}^{\mathcal{F}} \stackrel{\mathrm{D}}{\rightarrow} \mathrm{I}_{\mathcal{F}}$ pointwise and uniformly, as $\mathrm{n} \rightarrow \infty$, when $\mathrm{f} \in \mathrm{C}_{\mathcal{F}}^{\mathrm{U}}\left(\mathbb{R}^{\mathrm{d}}\right)$.

Proof. We observe that

$$
\begin{gathered}
D\left(\left(M_{n}^{\mathcal{F}}(f)\right)(\vec{x}), f(\vec{x})\right)= \\
\sup _{r \in[0,1]} \max \left\{\left|\left(M_{n}^{\mathcal{F}}(f)\right)_{-}^{(r)}(\vec{x})-f_{-}^{(r)}(\vec{x})\right|,\left|\left(M_{n}^{\mathcal{F}}(f)\right)_{+}^{(r)}(\vec{x})-f_{+}^{(r)}(\vec{x})\right|\right\} \stackrel{\frac{32}{=}}{=} \\
\sup _{r \in[0,1]} \max \left\{\left|\left(M_{n}\left(f_{-}^{(r)}\right)\right)(\vec{x})-f_{-}^{(r)}(\vec{x})\right|,\left|\left(M_{n}\left(f_{+}^{(r)}\right)\right)(\vec{x})-f_{+}^{(r)}(\vec{x})\right|\right\} \stackrel{\frac{26}{\leq}}{\leq} \\
\sup _{r \in[0,1]} \max \left\{\omega_{1}\left(f_{-}^{(r)}, \frac{T^{*}}{n^{1-\alpha}}\right), \omega_{1}\left(f_{+}^{(r)}, \frac{T^{*}}{n^{1-\alpha}}\right)\right\} \stackrel{7}{=} \omega_{1}^{(\mathcal{F})}\left(f, \frac{T^{*}}{n^{1-\alpha}}\right),
\end{gathered}
$$

proving the claim.

We continue with

Theorem 27. Let $\vec{\chi} \in \mathbb{R}^{\mathrm{d}}, \mathrm{f} \in \mathrm{C}_{\mathcal{F}}^{\mathrm{N}}\left(\mathbb{R}^{\mathrm{d}}\right), \mathrm{N} \in \mathbb{N}$, such that all of its fuzzy partial derivatives $\mathrm{f}_{\widetilde{\alpha}}$ of order $\mathrm{N}, \widetilde{\alpha}:|\widetilde{\alpha}|=\mathrm{N}$, are fuzzy uniformly continuous or fuzzy continuous and fuzzy bounded. Then

$$
\mathrm{D}\left(\left(\mathrm{M}_{\mathrm{n}}^{\mathcal{F}}(\mathrm{f})\right)(\vec{x}), f(\vec{x})\right) \leq
$$




$$
\begin{gathered}
\left\{\sum_{j=1}^{N} \frac{\left(T^{*}\right)^{j}}{j ! n^{j(1-\alpha)}}\left[\left(\sum_{i=1}^{d} D\left(\frac{\partial}{\partial x_{i}}, \widetilde{0}\right)\right)^{j} f(\vec{x})\right]\right\} \\
+\frac{\left(T^{*}\right)^{N} d^{N}}{N ! n^{N(1-\alpha)}} \max _{\tilde{\alpha}:|\widetilde{\alpha}|=N} \omega_{1}^{(\mathcal{F})}\left(f_{\widetilde{\alpha}}, \frac{T^{*}}{n^{1-\alpha}}\right) .
\end{gathered}
$$

As $\mathrm{n} \rightarrow \infty$, we get $\mathrm{D}\left(\left(\mathrm{M}_{\mathrm{n}}^{\mathcal{F}}(\mathrm{f})\right)(\overrightarrow{\mathrm{x}}), \mathrm{f}(\overrightarrow{\mathrm{x}})\right) \rightarrow 0$ pointwise with rates.

Proof. As before we have

$$
\begin{aligned}
& \mathrm{D}\left(\left(\mathrm{M}_{\mathrm{n}}^{\mathcal{F}}(\mathrm{f})\right)(\vec{x}), \mathrm{f}(\overrightarrow{\mathrm{x}})\right) \stackrel{32}{=} \\
& \sup _{r \in[0,1]} \max \left\{\left|\left(M_{n}\left(f_{-}^{(r)}\right)\right)(\vec{x})-f_{-}^{(r)}(\vec{x})\right|,\left|\left(M_{n}\left(f_{+}^{(r)}\right)\right)(\vec{x})-f_{+}^{(r)}(\vec{x})\right|\right\} \stackrel{27}{\leq} \\
& \sup _{r \in[0,1]} \max \left\{\left\{\sum_{j=1}^{N} \frac{\left(T^{*}\right)^{j}}{j ! n^{j(1-\alpha)}}\left[\left(\sum_{i=1}^{d}\left|\frac{\partial}{\partial x_{i}}\right|\right)^{j} f_{-}^{(r)}(\vec{x})\right]\right\}\right. \\
& +\frac{\left(T^{*}\right)^{N} d^{N}}{N ! n^{N(1-\alpha)}} \max _{\widetilde{\alpha}:|\widetilde{\alpha}|=N} \omega_{1}\left(\left(f_{-}^{(r)}\right)_{\widetilde{\alpha}}, \frac{T^{*}}{n^{1-\alpha}}\right), \\
& \left\{\sum_{j=1}^{N} \frac{\left(T^{*}\right)^{j}}{j ! n^{j(1-\alpha)}}\left[\left(\sum_{i=1}^{d}\left|\frac{\partial}{\partial x_{i}}\right|\right)^{j} f_{+}^{(r)}(\vec{x})\right]\right\} \\
& \left.+\frac{\left(T^{*}\right)^{N} d^{N}}{N ! n^{N(1-\alpha)}} \max _{\widetilde{\alpha}:|\widetilde{\alpha}|=N} \omega_{1}\left(\left(f_{+}^{(r)}\right)_{\widetilde{\alpha}}, \frac{T^{*}}{n^{1-\alpha}}\right)\right\} \stackrel{\sqrt[3]{3}}{\leq} \sum_{j=1}^{N} \frac{\left(T^{*}\right)^{j}}{j ! n^{j(1-\alpha)}} . \\
& \sup _{r \in[0,1]} \max \left\{\left(\left(\sum_{i=1}^{d}\left|\frac{\partial}{\partial x_{i}}\right|\right)^{j} f_{-}^{(r)}(\vec{x})\right),\left(\left(\sum_{i=1}^{d}\left|\frac{\partial}{\partial x_{i}}\right|\right)^{j} f_{+}^{(r)}(\vec{x})\right)\right\}+ \\
& \frac{\left(T^{*}\right)^{N} d^{N}}{N ! n^{N(1-\alpha)}} \max _{\widetilde{\alpha}:|\widetilde{\alpha}|=N_{r} \in[0,1]} \sup _{\max }\left\{\omega_{1}\left(\left(f_{-}^{(r)}\right)_{\widetilde{\alpha}}, \frac{T^{*}}{n^{1-\alpha}}\right), \omega_{1}\left(\left(f_{+}^{(r)}\right)_{\widetilde{\alpha}}, \frac{T^{*}}{n^{1-\alpha}}\right)\right\} \\
& \text { (by (3), (77), (12), (18)) }\left\{\sum_{j=1}^{N} \frac{\left(T^{*}\right)^{j}}{j ! n^{j(1-\alpha)}}\left[\left(\sum_{i=1}^{d} \mathrm{D}\left(\frac{\partial}{\partial x_{i}}, \widetilde{0}\right)\right)^{j} f(\vec{x})\right]\right\}+ \\
& \frac{\left(T^{*}\right)^{N} d^{N}}{N ! n^{N(1-\alpha)}} \max _{\tilde{\alpha}:|\widetilde{\alpha}|=N} \omega_{1}^{(\mathcal{F})}\left(f_{\widetilde{\alpha}}, \frac{T^{*}}{n^{1-\alpha}}\right)
\end{aligned}
$$

proving the claim. 


\section{Main Results - The fuzzy multivariate "normalized squash- ing type operators" and their fuzzy convergence to the fuzzy unit with rates}

We give the following definition

Definition 28. Let the nonnegative function $S: \mathbb{R}^{\mathrm{d}} \rightarrow \mathbb{R}, \mathrm{d} \geq 1, \mathrm{~S}$ has compact support $\mathcal{B}:=$ $\prod_{i=1}^{\mathrm{d}}\left[-\mathrm{T}_{i}, \mathrm{~T}_{i}\right], \mathrm{T}_{i}>0$ and is nondecreasing there for each coordinate. $\mathrm{S}$ can be continuous only on either $\prod_{i=1}^{\mathrm{d}}\left(-\infty, \mathrm{T}_{i}\right]$ or $\mathcal{B}$ and can have jump discontinuities. We call $\mathrm{S}$ the multivariate "squashing function" (see also [10]).

Let $f: \mathbb{R}^{\mathrm{d}} \rightarrow \mathbb{R}_{\mathcal{F}}$ be either fuzzy uniformly continuous or fuzzy continuous and fuzzy bounded function.

For $\vec{\chi} \in \mathbb{R}^{\mathrm{d}}$, we define the fuzzy multivariate " normalized squashing type operator",

$$
\begin{gathered}
L_{n}^{\mathcal{F}}(f)(\vec{x}):= \\
\frac{\sum_{k_{1}=-n^{2}}^{n^{2} *} \sum_{k_{d}=-n^{2}}^{n^{2} *}\left(\frac{k_{1}}{n}, \ldots \frac{k_{d}}{n}\right) \odot S\left(n^{1-\alpha}\left(x_{1}-\frac{k_{1}}{n}\right), \ldots, n^{1-\alpha}\left(x_{d}-\frac{k_{d}}{n}\right)\right)}{W(\vec{x})},
\end{gathered}
$$

where $0<\alpha<1$ and $n \in \mathbb{N}$ :

$$
\mathrm{n} \geq \max _{i \in\{1, \ldots, \mathrm{d}\}}\left\{\mathrm{T}_{i}+\left|x_{i}\right|, \mathrm{T}_{i}^{-\frac{1}{\alpha}}\right\}
$$

and

$$
W(\vec{x}):=\sum_{k_{1}=-n^{2}}^{n^{2} *} \ldots \sum_{k_{d}=-n^{2}}^{n^{2} *} S\left(n^{1-\alpha}\left(x_{1}-\frac{k_{1}}{n}\right), \ldots, n^{1-\alpha}\left(x_{d}-\frac{k_{d}}{n}\right)\right) .
$$

It is clear that

$$
\left(L_{n}^{\mathcal{F}}(f)\right)(\vec{x}):=\sum_{\vec{k}=\left\lceil n \vec{x}-\vec{T} n^{\alpha}\right\rceil}^{\left[n \vec{x}+\vec{T} n^{\alpha}\right] *} \frac{f\left(\frac{\vec{k}}{n}\right) \odot S\left(n^{1-\alpha}\left(\vec{x}-\frac{\vec{k}}{n}\right)\right)}{\Phi(\vec{x})}
$$

where

$$
\Phi(\vec{x}):=\sum_{\vec{k}=\left\lceil n \vec{x}-\vec{T} n^{\alpha}\right\rceil}^{\left[n \vec{x}+\vec{T} n^{\alpha}\right]} S\left(n^{1-\alpha}\left(\vec{x}-\frac{\vec{k}}{n}\right)\right)
$$

Here, we study the D-metric pointwise convergence with rates of $\left(L_{\mathfrak{n}}^{\mathcal{F}}(f)\right)(\vec{x}) \rightarrow f(\vec{x})$, as $\mathrm{n} \rightarrow+\infty, \vec{x} \in \mathbb{R}^{\mathrm{d}}$.

This is given first by the next result. 
Theorem 29. Under the above terms and asumptions, we find that

$$
\mathrm{D}\left(\left(\mathrm{L}_{\mathrm{n}}^{\mathcal{F}}(\mathrm{f})\right)(\overrightarrow{\mathrm{x}}), \mathrm{f}(\overrightarrow{\mathrm{x}})\right) \leq \omega_{1}^{(\mathcal{F})}\left(\mathrm{f}, \frac{\mathrm{T}^{*}}{\mathrm{n}^{1-\alpha}}\right) .
$$

Notice that 43) gives $\mathrm{L}_{\mathfrak{n}}^{\mathcal{F}} \stackrel{\mathrm{D}}{\rightarrow} \mathrm{I}_{\mathcal{F}}$ pointwise and uniformly, as $\mathrm{n} \rightarrow \infty$, when $\mathrm{f} \in \mathrm{C}_{\mathcal{F}}^{\mathrm{u}}\left(\mathbb{R}^{\mathrm{d}}\right)$.

Proof. Similar to (33).

We also give

Theorem 30. Let $\vec{x} \in \mathbb{R}^{\mathrm{d}}, \mathrm{f} \in \mathrm{C}_{\mathcal{F}}\left(\mathbb{R}^{\mathrm{d}}\right), \mathrm{N} \in \mathbb{N}$, such that all of its fuzzy partial derivatives $\mathbf{f}_{\widetilde{\alpha}}$ of order $\mathrm{N}, \widetilde{\alpha}:|\widetilde{\alpha}|=\mathrm{N}$, are fuzzy uniformly continuous or fuzzy continuous and fuzzy bounded. Then

$$
\begin{gathered}
\mathrm{D}\left(\left(\mathrm{L}_{\mathrm{n}}^{\mathcal{F}}(\mathrm{f})\right)(\vec{x}), f(\vec{x})\right) \leq \\
\left\{\sum_{j=1}^{N} \frac{\left(T^{*}\right)^{j}}{j ! n^{j(1-\alpha)}}\left[\left(\sum_{i=1}^{d} \mathrm{D}\left(\frac{\partial}{\partial x_{i}}, \widetilde{0}\right)\right)^{j} f(\vec{x})\right]\right\} \\
+\frac{\left(T^{*}\right)^{N} d^{N}}{N ! n^{N(1-\alpha)}} \max _{\widetilde{\alpha}:|\widetilde{\alpha}|=N} \omega_{1}^{(\mathcal{F})}\left(f_{\widetilde{\alpha}}, \frac{T^{*}}{n^{1-\alpha}}\right) .
\end{gathered}
$$

Inequality (44) gives us with rates the poitwise convergence of $\mathrm{D}\left(\left(\mathrm{L}_{n}^{\mathcal{F}}(f)\right)(\vec{x}), f(\vec{x})\right) \rightarrow 0$ over $\mathbb{R}^{\mathrm{d}}$, as $\mathrm{n} \rightarrow \infty$.

Proof. Similar to (34).

Received: November 2012. Accepted: May 2014.

\section{References}

[1] G.A. Anastassiou, Rate of convergence of some neural network operators to the unit-univariate case, Journal of Mathematical Analysis and Application, Vol. 212 (1997), 237-262.

[2] G.A. Anastassiou, Rate of Convergence of some Multivariate Neural Network Operators to the Unit, Computers and Mathematics, 40(2000), 1-19.

[3] G.A. Anastassiou, Quantitative Approximation, Chapmann and Hall/CRC, Boca Raton, New York, 2001.

[4] G.A. Anastassiou, Higher order Fuzzy Approximation by Fuzzy Wavelet type and Neural Network Operators, Computers and Mathematics, 48(2004), 1387-1401. 
[5] G.A. Anastassiou, Fuzzy Approximation by Fuzzy Convolution type Operators, Computers and Mathematics, 48(2004), 1369-1386.

[6] G.A. Anastassiou, Higher order Fuzzy Korovkin Theory via inequalities, Communications in Applied Analysis, 10(2006), No. 2, 359-392.

[7] G.A. Anastassiou, Fuzzy Korovkin Theorems and Inequalities, Journal of Fuzzy Mathematics, 15(2007), No. 1, 169-205.

[8] G.A. Anastassiou, Higher order multivariate fuzzy approximation by multivariate fuzzy wavelet type and neural network operators, J. of Fuzzy Mathematics, 19(2011), no. 3, 601-618.

[9] G.A. Anastassiou, Rate of convergence of some multivariate neural network operators to the unit,revisited, J. of Computational Analysis and Application, to appear 2013.

[10] P. Cardaliaguet, G. Euvrard, Approximation of a function and its derivative with a neural network, Neural Networks 5(1992), 207-220.

[11] R. Goetschel Jr., W. Voxman, Elementary fuzzy calculus, Fuzzy Sets and Systems, 18(1986), 31-43.

[12] O. Kaleva, Fuzzy differential equations, Fuzzy Sets and Systems, 24(1987), 301-317.

[13] Y.K. Kim, B.M. Ghil, Integrals of fuzzy-number-valued functions, Fuzzy Sets and Systems, 86(1997), 213-222.

[14] C. Wu, Z. Gong, On Henstock integral of fuzzy-number-valued functions (I), Fuzzy Sets and Systems, 120, No. 3, (2001), 523-532.

[15] C. Wu, M. Ma, On embedding problem of fuzzy numer spaces: Part 1, Fuzzy Sets and Systems, 44 (1991), 33-38. 